\title{
Caesarean scar site complete molar pregnancy
}

\author{
Kaluarachchi C I, Tissera A J, Gananatha Karunarathna S M G
}

\section{Summary}

A 40 year old multiparous asymptomatic woman presented at 9+ weeks with a finding of rising serum beta-HCG over $3500 \mathrm{IU}$ without ultrasonically detectable intra-uterine pregnancy. First diagnostic laparoscopy was performed on 3rd day of admission and found to be normal. Because of rising serum beta-HCG second laparoscopy was performed and it was converted to a laparotomy as a caesarean scar site pregnancy was suspected. Sub-total hysterectomy was performed in order to remove suspected caesarean scar molar pregnancy. Patient recovered completely with normal beta-HCG on 20th post day. Pieces of tissue obtained from between the anterior abdominal wall and the previous caesarean section scar revealed diagnosis of complete hydatidiform mole.

Background: Implantation at the caesarian scar site can lead to first trimester miscarriages, placenta previa, placenta accreta or ectopic pregnancy. While caesarean scar site ectopic pregnancy is a rare occurrence; caesarian scar site molar pregnancy is extremely rare. We report this case to highlight the importance of high index of suspicion and proper monitoring in diagnosing this condition.

Key words: caesarean section, gestational trophoblastic disease, hysterectomy

$\mathrm{A}$ 40 year old previously healthy mother of three children presented to the antenatal clinic for a routine booking visit. She has had two past caesarian sections, the first one being due to a twin pregnancy. The second section had been performed 7 years ago. She has had depot medoxy progesterone acetate for contraception for two years after the last pregnancy, following which she has had normal regular menstrual bleeding. At the booking visit it was found to be an unplanned pregnancy with a period of amenorrhea of 9 weeks +5 days. She did not have abdominal pain, per vaginal bleeding or any other significant complain. A routine trans-

Obstetrics and Gynaecology Unit, Sri Jayewardenepura General Hospital, Colombo, Sri Lanka.

Correspondence: S M G Karunarathna

E-mail: madhava63@hotmail.com vaginal ultra sound scan was done, in which; there was no intra uterine pregnancy (IUP), endometrial thickness was $13 \mathrm{~mm}$, there were no adenexial masses or free fluid.

She was admitted for further management of pregnancy of unknown location and serum $\beta$ hCG was monitored. The first $\beta$ hCG level came as $6743 \mathrm{mIU} / \mathrm{ml}$ and it rose to $8783 \mathrm{mIU} /$ $\mathrm{ml}$ after 48 hours. Since there was still no IUP the first diagnostic laparoscopy was performed on the 3rd day after admission. During the laparoscopy the uterus was found to be adhered to the anterior abdominal wall and there were bowel adhesions. However bilateral tubes and ovaries were normal, there was no free fluid and no evidence of an ectopic pregnancy. However the serum $\beta$ hCG level was continuously monitored and was found to be rising. By the 12th day after the laparoscopy, it had risen to $39248 \mathrm{mIU} / \mathrm{ml}$. Thus a second diagnostic laparoscopy was performed, which was converted to a laparotomy as a caesarean scar site pregnancy was suspected.

During the laparotomy thick adhesions were found between the anterior abdominal wall and the uterus. While dissecting the uterus from the anterior abdominal wall the uterine cavity was opened into, and products of conception, suspicious of a molar pregnancy, were found between the anterior abdominal wall and the previous caesarian section scar. Subtotal hysterectomy was performed while preserving ovaries. After the surgery the serum $\beta$ hCG level dropped and on the 20th day post op it was 39 $\mathrm{mIU} / \mathrm{ml}$.

The histopathology of the uterus and tubes revealed a secretory endometrium in a decidualized stroma with histologically unremarkable tubes. However the pieces of tissue obtained from between the anterior abdominal wall and the previous caesarian section scar revealed enlarged chorionic villi with central cisternal changes and trophoblastic proliferation, which confirmed the diagnosis of complete hydatidiform mole.

She is being followed up with regular clinical assessments and serum $\beta$ hCG measurements to exclude development of post-molar gestational trophoblastic neoplasia.

\section{Discussion}

Gestational trophoblastic disease is a spectrum of diseases including hydatidiform moles, invasive moles, gestational choriocarcinomas, and placental site trophoblastic tumors. While cytogenesis, pathology, and clinical presentation differ between partial mole and complete mole, the management is almost similar. However post-molar gestational trophoblastic neoplasia (GTN) and metastatic GTN is diagnosed more frequently after complete moles than partial moles ${ }^{3}$. 


\section{Figure 1. Uterus adhered to the anterior abdominal Figure 2. Molar tissue between the uterus and the wall, with normal pouch of Douglas. anterior abdominal wall.}
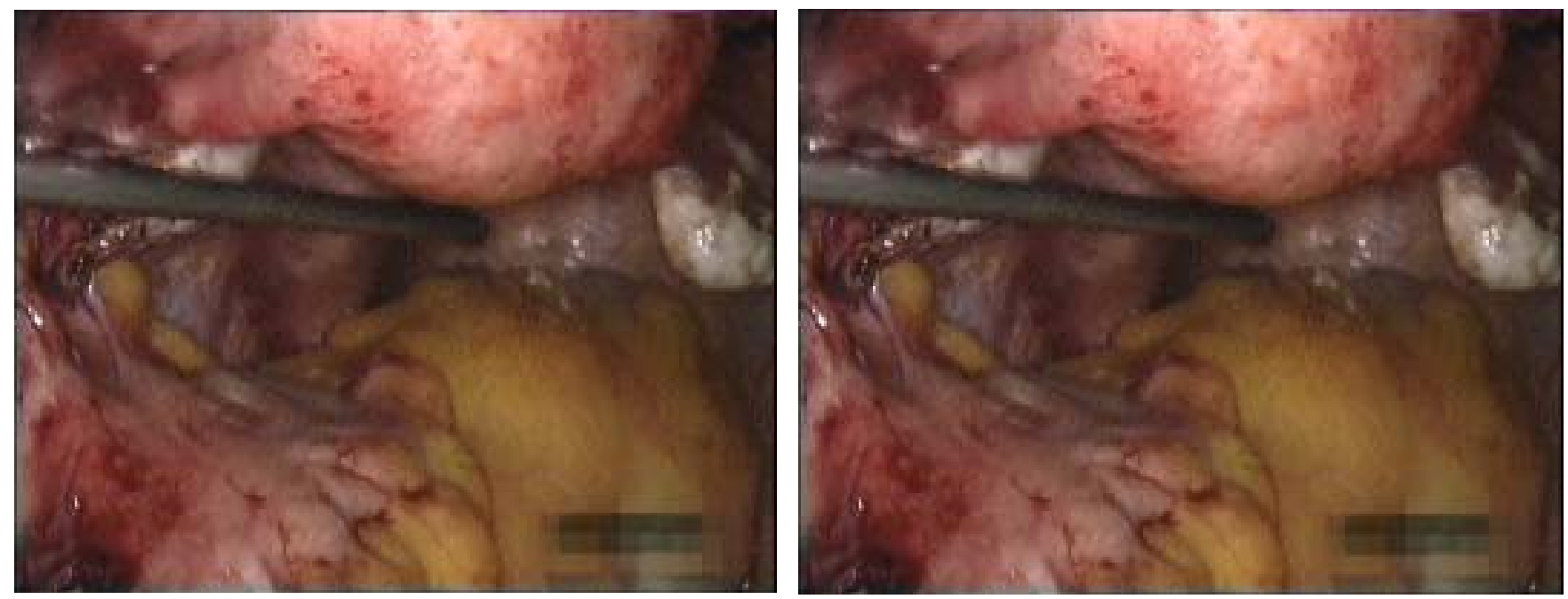

Caesarean site molar pregnancy is extremely rare and carries a high risk of uterine rupture and uncontrollable hemorrhage. While few cases of caesarian scar site partial molar pregnancies have been reported worldwide, current literature does not reveal any cases of caesarian scar site complete molar pregnancy.

Almost all the reported cases of caesarean site molar pregnancy describes patients presenting with symptoms of early pregnancy complications such as per-vaginal bleeding ${ }^{4}$, abdominal pain, persistent spotting after uterine evacuation ${ }^{2}$ etc. However this case is unique as the patient was asymptomatic on admission and throughout the hospital stay. This also highlights the importance of early pregnancy ultrasound scan especially in high risk patients. Advanced maternal age was a significant risk factor in this patient as maternal age above 40 years is known to be associated with 11-fold increase in the relative risk of complete molar pregnancy. Trans-vaginal ultrasound scan plays a major role in diagnosing this condition. Jennifer et al describes; complex lesion with cystic spaces and increased vascularity at the anterior lower uterine wall with a thin layer of

\section{Figure 3. Enlarged chorionic villi with central cisternal changes characteristic of complete hydatidiform mole.}

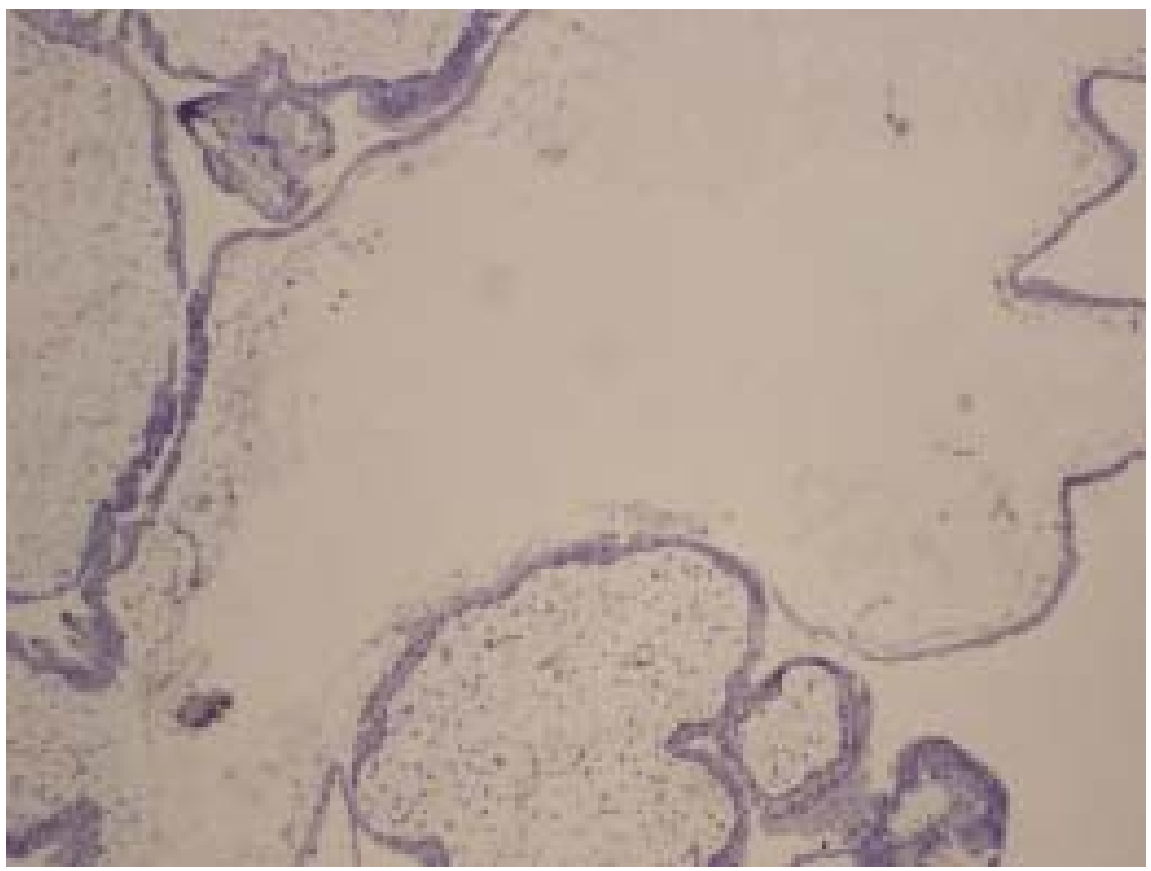

overlying myometrium and no evidence of an intrauterine gestational sac as ultrasound markers of caesarean site molar pregnancy ${ }^{2}$. However a negative ultrasound scan does not rule out the possibility and in this patient high index of suspicion even in the absence of ultra sound findings lead to the ultimate diagnosis.

Systemic or local methotrexate therapy, suction curettage and hysterectomy have been successfully used in the 
management of caesarian scar site ectopic pregnancy. Michener et al describes a case series where five cases had been successfully treated with systemic methotrexate therapy, two cases had been successfully treated with dilatation and suction curettage and only three cases had needed primary hysterectomy due to haemodynamic instability or due to patient preference of definitive surgery ${ }^{6}$. However in the same case series local methotrexate therapy had failed in a patient with caesarian scar site partial molar pregnancy and she had and later presented with life-threatening haemorrhage and required an emergency hysterectomy. There are no clear guidelines as to the management of caesarian scar site molar pregnancy. The current literature reveals ultra-sound guided suction evacuation to be the most widely used method and the most successful ${ }^{2,5}$. However in this particular patient, since the condition was not detected prior to the surgery and as the family was complete we had to resort to sub-total hysterectomy.

\section{REFERENCES}

1. Jurkovic D, Hillaby K, Woelfer B, Lawrence A, Salim R, Elson CJ. First-trimester diagnosis and management of pregnancies implanted into the lower uterine segment Cesarean section scar. Ultrasound in Obstetrics and Gynecology 2003; 21:220-7.

2. Ko JKY, Wan HL, Ngu SF, Cheung VYT, $\mathrm{Ng}$ EHY. Cesarean Scar Molar Pregnancy. Obstetrics and Gynaecology 2012; 119(2)2: 449-51.

3. John T. Soper. Gestational Trophoblastic Disease. Obstetrics and Gynecology 2006; 108(1): 176-87.
4. Wu CF, Hsu CY, Chen CP. Ectopic Molar Pregnancy in a Cesarean Scar. Taiwanese Journal of Obstetrics and Gynecology 2006; 45(4): 3435.

5. Jin FS, Ding DC, Wu GJ, Hwang KS. Molar Pregnancy in a Cesarean Section Scar of Uterus. Journal of Medical Sciences 2011; 31(4): 173-6.

6. Michener C, Dickinson JE. Caesarean Scar Ectopic Pregnancy: A Single Centre Case Series. Australian and New Zealand Journal of Obstetrics and Gynaecology. 2009; (49): 451-5.

7. Tan G, Chong YS, Biswas A. Caesarean Scar Pregnancy: A Diagnosis to Consider Carefully in Patients with Risk Factors. Annals Academy of Medicine. 2005; 34(2): 216-19.

8. Potdar N, Navti OB, McParland P, Khare $M$, Elson J. Diagnosis and management of ectopic molar Caesarean scar pregnancy. Ultrasound in Obstetrics and Gynecology $2010 ; 36(1): 231$. 